\title{
Infinitely many homoclinic orbits for a class of discrete Hamiltonian systems
}

\section{Xianhua Tang* and Jing Chen}

"Correspondence:
tangxh@mail.csu.edu.cn
School of Mathematics and
Statistics, Central South University,
Changsha, Hunan 410083, P.R. China

Changsha, Hunan 410083, P.R. China

\begin{abstract}
In the present paper, we deal with the existence of infinitely many homoclinic solutions for the second-order self-adjoint discrete Hamiltonian system

$$
\triangle[p(n) \Delta u(n-1)]-L(n) u(n)+\nabla W(n, u(n))=0,
$$

where $p(n)$ and $L(n)$ are $\mathcal{N} \times \mathcal{N}$ real symmetric matrices for all $n \in \mathbb{Z}$, and $p(n)$ is always positive definite. Under the assumptions that $L(n)$ is allowed to be sign-changing and satisfies

$$
\lim _{|n| \rightarrow+\infty} \inf _{|x|=1}(L(n) x, x)=\infty
$$

$W(n, x)$ is of indefinite sign and superquadratic as $|x| \rightarrow+\infty$, we establish several existence criteria to guarantee that the above system has infinitely many homoclinic solutions.
\end{abstract}

MSC: $39 \mathrm{~A} 11 ; 58 \mathrm{E} 05 ; 70 \mathrm{HO} 05$

Keywords: homoclinic solution; discrete Hamiltonian system; superquadratic; critical point

\section{Introduction}

Consider the second-order self-adjoint discrete Hamiltonian system

$$
\triangle[p(n) \triangle u(n-1)]-L(n) u(n)+\nabla W(n, u(n))=0,
$$

where $n \in \mathbb{Z}, u \in \mathbb{R}^{\mathcal{N}}, \Delta u(n)=u(n+1)-u(n)$ is the forward difference operator, $p, L: \mathbb{Z} \rightarrow$ $\mathbb{R}^{\mathcal{N} \times \mathcal{N}}$ and $W: \mathbb{Z} \times \mathbb{R}^{\mathcal{N}} \rightarrow \mathbb{R}, W(n, x)$ is continuously differentiable in $x$ for every $n \in \mathbb{Z}$. In general, system (1.1) may be regarded as a discrete analogue of the following second-order Hamiltonian system

$$
\left[p(t) u^{\prime}(t)\right]^{\prime}-L(t) u(t)+\nabla W(t, u(t))=0
$$

Moreover, system (1.1) has applications as is shown in the monographs [1,2]. In the past 40 years, system (1.2) has been widely investigated, see [3-9] and references therein. System (1.2) is the special form of the Emden-Fowler equation, appearing in the study of astrophysics, gas dynamics, fluid mechanics, relativistic mechanics, nuclear physics and chem-

\section{Springer}

(c) 2013 Tang and Chen; licensee Springer. This is an Open Access article distributed under the terms of the Creative Commons Attribution License (http://creativecommons.org/licenses/by/2.0), which permits unrestricted use, distribution, and reproduction in any medium, provided the original work is properly cited. 
ically reacting systems, and many well-known results concerning properties of solutions of (1.2) are collected in [10].

As usual, we say that a solution $u(n)$ of system (1.1) is homoclinic (to 0$)$ if $u(n) \rightarrow 0$ as $n \rightarrow \pm \infty$. In addition, if $u(n) \not \equiv 0$, then $u(n)$ is called a nontrivial homoclinic solution.

The existence and the multiplicity of homoclinic solutions of system (1.1) or its special forms have been investigated by many authors. Papers [11-13] deal with the periodic case, where $p, L$ and $W$ are $N$-periodic in $n$. If the periodicity is lost, the case is quite different from the ones just described, because of lack of compactness of the Sobolev embedding. In this case, either a coercivity condition on $L$ are required to be satisfied, see [14-19], or $W(n, x)$ can be dominated by a summable function, see $[5,13]$. In the above-mentioned papers, except [16], $L$ was always required to be positive definite. Meanwhile, $W$ was always assumed to be superquadratic as $x \rightarrow 0$ uniformly for $n \in \mathbb{Z}$, i.e.,

(W0) $\lim _{|x| \rightarrow 0} \frac{W(n, x)}{|x|^{2}}=0$ uniformly for $n \in \mathbb{Z}$.

In addition, $W(n, x)$ is subquadratic as $|x| \rightarrow \infty$ in [17, 20], while $W(n, x)$ is superquadratic in $[11-16,18,19,21]$. Moreover, in the superquadratic case, except [5], the well-known global Ambrosetti-Rabinowitz superquadratic condition was always assumed:

(AR) there exists $\mu>2$ such that

$$
0<\mu W(n, x) \leq(\nabla W(n, x), x), \quad \forall(n, x) \in \mathbb{Z} \times \mathbb{R}^{\mathcal{N}} \backslash\{0\},
$$

where and in the sequel, $(\cdot, \cdot)$ denotes the standard inner product in $\mathbb{R}^{\mathcal{N}}$, and $|\cdot|$ is the induced norm.

However, in mathematical physics, it is of frequent occurrence in a system like (1.1) that the global positive definiteness of $L(n)$ is not satisfied. This is seen, for example, $L(n)=$ $\left[l_{+}(n)-l_{-}(n)\right] I_{\mathcal{N}}$, where $l_{+}(n) \geq 0, l_{+}(n) \rightarrow+\infty$ as $|n| \rightarrow+\infty$, and $l_{-}(n)$ is bounded, or $L(n)=l(n) I_{\mathcal{N}}, l(n)$ is a polynomial of degree $2 m$ with the property that the coefficient of the leading term is positive.

In this paper, we are interested in the case when $L(n)$ is not global positive definite and satisfies the following assumption.

(L) $L(n)$ is an $\mathcal{N} \times \mathcal{N}$ real symmetric matrix for all $n \in \mathbb{Z}$ and the smallest eigenvalue of $L(n) \rightarrow \infty$ as $|n| \rightarrow \infty$, i.e.,

$$
\lim _{|n| \rightarrow \infty}\left[\inf _{|x|=1}(L(n) x, x)\right]=\infty .
$$

Under assumption $(\mathrm{L})$ above, we will use the symmetric mountain pass theorem to study the existence of infinitely many homoclinic solutions for (1.1) in the case, where $W$ satisfies the following weaker assumptions than (W0) as $x \rightarrow 0$ and (AR) as $|x| \rightarrow \infty$.

(W1) $W(n, x)$ is continuously differentiable in $x$ for every $n \in \mathbb{Z}, W(n, 0) \equiv 0$, and there exist constants $c_{0}>0$ and $R_{0}>0$ such that

$$
|\nabla W(n, x)| \leq c_{0}|x|, \quad \forall(n, x) \in \mathbb{Z} \times \mathbb{R}^{\mathcal{N}},|x| \leq R_{0} ;
$$

(W2) $\lim _{|x| \rightarrow \infty} \frac{|W(n, x)|}{|x|^{2}}=\infty$ for all $n \in \mathbb{Z}$, and

$$
W(n, x) \geq 0, \quad \forall(n, x) \in \mathbb{Z} \times \mathbb{R}^{\mathcal{N}},|x| \geq R_{0}
$$


(W3) $W(n,-x)=W(n, x), \forall(n, x) \in \mathbb{Z} \times \mathbb{R}^{\mathcal{N}}$;

(W4) $\widetilde{W}(n, x):=\frac{1}{2}(\nabla W(n, x), x)-W(n, x) \geq g(n), \forall(n, x) \in \mathbb{Z} \times \mathbb{R}^{\mathcal{N}}$, where $|g| \in l^{1}(\mathbb{Z}, \mathbb{R})$, and there exists $c_{1}>0$ such that

$$
|W(n, x)| \leq c_{1}|x|^{2} \widetilde{W}(n, x), \quad \forall(n, x) \in \mathbb{Z} \times \mathbb{R}^{\mathcal{N}},|x| \geq R_{0} ;
$$

(W5) there exist $\mu>2$ and $\varrho>0$ such that

$$
\mu W(n, x) \leq(\nabla W(n, x), x)+\varrho|x|^{2}, \quad \forall(n, x) \in \mathbb{Z} \times \mathbb{R}^{\mathcal{N}}
$$

(W6) there exists a $\mu>2$ such that

$$
\mu W(n, x) \leq(\nabla W(n, x), x), \quad \forall(n, x) \in \mathbb{Z} \times \mathbb{R}^{\mathcal{N}},|x| \geq R_{0}
$$

Now, we are ready to state the main results of this paper.

Theorem 1.1 Assume that $p(n)$ is an $\mathcal{N} \times \mathcal{N}$ real symmetric positive definite matrix for all $n \in \mathbb{Z}, L$ and $W$ satisfy (L), (W1), (W2), (W3) and (W4). Then system (1.1) possesses infinitely many nontrivial homoclinic solutions.

Theorem 1.2 Assume that $p(n)$ is an $\mathcal{N} \times \mathcal{N}$ real symmetric positive definite matrix for all $n \in \mathbb{Z}, L$ and W satisfy (L), (W1), (W2), (W3) and (W5). Then system (1.1) possesses infinitely many nontrivial homoclinic solutions.

It is easy to check that (W1) and (W6) imply (W5). Thus, we have the following corollary.

Corollary 1.3 Assume that $p(n)$ is an $\mathcal{N} \times \mathcal{N}$ real symmetric positive definite matrix for all $n \in \mathbb{Z}, L$ and $W$ satisfy (L), (W1), (W2), (W3) and (W6). Then system (1.1) possesses infinitely many nontrivial homoclinic solutions.

Remark 1.4 In our theorems, $L(n)$ is allowed to be sign-changing, for example,

$$
L(n)=\left(n^{2}-10\right) I_{\mathcal{N}}
$$

Moreover, $W(n, x)$ is also allowed to be sign-changing. Even if $W(n, x) \geq 0$, assumptions (W2), (W4), (W5) and (W6) are weaker than the superquadratic conditions, obtained in the aforementioned references. It is easy to check that the following functions $W$ satisfy (W1), (W2), (W3) and (W4) or (W6):

$$
\begin{aligned}
& W(n, x)=\left(1+\sin ^{2} n\right)|x|^{2} \ln \left(\frac{1}{2}+|x|\right), \\
& W(n, x)=e^{\left(1+n^{2}\right)|x|^{2} /\left(2+n^{2}\right)}-1, \\
& W(n, x)=\frac{1}{1+n^{2}}\left[4|x|^{5}+2|x|^{3} \sin |x|-4|x|^{2} \cos |x|\right]
\end{aligned}
$$

and

$$
W(n, x)=a(n) \sum_{i=1}^{m} b_{i}|x|^{\beta_{i}},
$$


where $b_{1}>0, b_{i} \in \mathbb{R}, i=2,3, \ldots, m, \beta_{1}>\beta_{2}>\cdots>\beta_{m} \geq 2$, and $0<\inf _{\mathbb{Z}} a \leq \sup _{\mathbb{Z}} a<\infty$. One can see that they do not satisfy (W0) or (AR).

\section{Preliminaries}

Throughout this section, we always assume that $p(n)$ is real symmetric positive definite matrix for all $n \in \mathbb{Z}$. Set

$$
l(n)=\inf _{x \in \mathbb{R}^{\mathcal{N}},|x|=1}(L(n) x, x),
$$

and make the following assumption on $L(n)$ instead of $(\mathrm{L})$ :

$\left(\mathrm{L}^{\prime}\right) L(n)$ is an $\mathcal{N} \times \mathcal{N}$ real symmetric matrix for all $n \in \mathbb{Z}, \lim _{|n| \rightarrow \infty} l(n)=\infty$, and

$$
(L(n) x, x) \geq|x|^{2}, \quad \forall(n, x) \in \mathbb{Z} \times \mathbb{R}^{\mathcal{N}} .
$$

Let

$$
\begin{aligned}
& S=\left\{\{u(n)\}_{n \in \mathbb{Z}}: u(n) \in \mathbb{R}^{\mathcal{N}}, n \in \mathbb{Z}\right\}, \\
& E=\left\{u \in S: \sum_{n \in \mathbb{Z}}[(p(n+1) \Delta u(n), \Delta u(n))+(L(n) u(n), u(n))]<+\infty\right\},
\end{aligned}
$$

and for $u, v \in E$, let

$$
\langle u, v\rangle=\sum_{n \in \mathbb{Z}}[(p(n+1) \Delta u(n), \Delta v(n))+(L(n) u(n), v(n))] .
$$

Then $E$ is a Hilbert space with the inner product above, and the corresponding norm is

$$
\|u\|=\left\{\sum_{n \in \mathbb{Z}}[(p(n+1) \Delta u(n), \Delta u(n))+(L(n) u(n), u(n))]\right\}^{1 / 2}, \quad u \in E .
$$

As usual, for $1 \leq q<+\infty$, set

$$
l^{q}\left(\mathbb{Z}, \mathbb{R}^{\mathcal{N}}\right)=\left\{u \in S: \sum_{n \in \mathbb{Z}}|u(n)|^{q}<+\infty\right\}
$$

and

$$
l^{\infty}\left(\mathbb{Z}, \mathbb{R}^{\mathcal{N}}\right)=\left\{u \in S: \sup _{n \in \mathbb{Z}}|u(n)|<+\infty\right\}
$$

and their norms are defined by

$$
\begin{aligned}
& \|u\|_{q}=\left(\sum_{n \in \mathbb{Z}}|u(n)|^{q}\right)^{1 / q}, \quad \forall u \in l^{q}\left(\mathbb{Z}, \mathbb{R}^{\mathcal{N}}\right) ; \\
& \|u\|_{\infty}=\sup _{n \in \mathbb{Z}}|u(n)|, \quad \forall u \in l^{\infty}\left(\mathbb{Z}, \mathbb{R}^{\mathcal{N}}\right),
\end{aligned}
$$


respectively. Evidently, $E$ is continuously embedded into $l^{q}\left(\mathbb{Z}, \mathbb{R}^{\mathcal{N}}\right)$ for $2 \leq q \leq+\infty$, i.e., there exists $\gamma_{q}>0$ such that

$$
\|u\|_{q} \leq \gamma_{q}\|u\|, \quad \forall u \in E
$$

Lemma 2.1 (Lin and Tang [15]) For $u \in E$, one has

$$
\|u\|_{\infty} \leq \frac{1}{\sqrt[4]{1+4 \alpha}}\|u\|
$$

where $\alpha=\inf \left\{(p(n) x, x): n \in \mathbb{Z}, x \in \mathbb{R}^{\mathcal{N}},|x|=1\right\}$.

Lemma 2.2 (Tang and Lin [17]) Suppose that L satisfies (L'). Then E is compactly embed$\operatorname{ded}$ in $l^{q}\left(\mathbb{Z}, \mathbb{R}^{\mathcal{N}}\right)$ for $2 \leq q<\infty$, and

$$
\|u\|_{q}^{q} \leq[1+4 \alpha]^{(2-q) / 4}\|u\|^{q}, \quad \forall u \in E
$$

Now, we define a functional $\Phi$ on $E$ by

$$
\Phi(u)=\frac{1}{2} \sum_{n \in \mathbb{Z}}[(p(n+1) \Delta u(n), \Delta u(n))+(L(n) u(n), u(n))]-\sum_{n \in \mathbb{Z}} W(n, u(n)) .
$$

For any $u \in E$, there exists an $N \in \mathbb{N}$ such that $|u(n)| \leq R_{0}$ for $|n| \geq N$. Hence, by (W1), one has

$$
|W(n, u(n))| \leq \frac{c_{0}}{2}|u(n)|^{2}, \quad|n| \geq N
$$

Consequently, under assumptions $\left(\mathrm{L}^{\prime}\right)$ and (W1), the functional $\Phi$ is of class $C^{1}(E, \mathbb{R})$. Moreover,

$$
\begin{aligned}
& \Phi(u)=\frac{1}{2}\|u\|^{2}-\sum_{n \in \mathbb{Z}} W(n, u(n)), \quad \forall u \in E, \\
& \left\langle\Phi^{\prime}(u), v\right\rangle=\langle u, v\rangle-\sum_{n \in \mathbb{Z}}(\nabla W(n, u(n)), v(n)), \quad \forall u, v \in E .
\end{aligned}
$$

Furthermore, the critical points of $\Phi$ in $E$ are solutions of system (1.1) with $u( \pm \infty)=0$, see $[14,16]$.

Lemma 2.3 Under assumptions (L'), (W1), (W2) and (W4), any sequence $\left\{u_{k}\right\} \subset E$ satisfying

$$
\Phi\left(u_{k}\right) \rightarrow c>0, \quad\left\langle\Phi^{\prime}\left(u_{k}\right), u_{k}\right\rangle \rightarrow 0,
$$

is bounded in $E$.

Proof To prove the boundedness of $\left\{u_{k}\right\}$, arguing by contradiction, suppose that $\left\|u_{k}\right\| \rightarrow$ $\infty$. Let $v_{k}=u_{k} /\left\|u_{k}\right\|$. Then $\left\|v_{k}\right\|=1$ and $\left\|v_{k}\right\|_{q} \leq \gamma_{q}\left\|v_{k}\right\|=\gamma_{q}$ for $2 \leq q \leq \infty$. Observe that 
for $k$ large

$$
c+1 \geq \Phi\left(u_{k}\right)-\frac{1}{2}\left\langle\Phi^{\prime}\left(u_{k}\right), u_{k}\right\rangle=\sum_{n \in \mathbb{Z}} \widetilde{W}\left(n, u_{k}(n)\right) .
$$

It follows from (2.7) and (2.9) that

$$
\frac{1}{2} \leq \limsup _{k \rightarrow \infty} \sum_{n \in \mathbb{Z}} \frac{\left|W\left(n, u_{k}(n)\right)\right|}{\left\|u_{k}\right\|^{2}}
$$

For $0 \leq a<b$, let

$$
\Omega_{k}(a, b)=\left\{n \in \mathbb{Z}: a \leq\left|u_{k}(n)\right|<b\right\} .
$$

Passing to a subsequence, we may assume that $v_{k} \rightarrow v$ in $E$, then by Lemma 2.2, $v_{k} \rightarrow v$ in $l^{q}\left(\mathbb{Z}, \mathbb{R}^{\mathcal{N}}\right), 2 \leq q<\infty$, and $v_{k}(n) \rightarrow v(n)$ for all $n \in \mathbb{Z}$.

If $v=0$, then $v_{k} \rightarrow 0$ in $l^{q}\left(\mathbb{Z}, \mathbb{R}^{\mathcal{N}}\right), 2 \leq q<\infty, v_{k}(n) \rightarrow 0$ for all $n \in \mathbb{Z}$. Hence, it follows from (W1) that

$$
\sum_{n \in \Omega_{k}\left(0, R_{0}\right)} \frac{\left|W\left(n, u_{k}(n)\right)\right|}{\left|u_{k}(n)\right|^{2}}\left|v_{k}(n)\right|^{2} \leq \frac{c_{0}}{2} \sum_{n \in \Omega_{k}\left(0, R_{0}\right)}\left|v_{k}(n)\right|^{2} \leq \frac{c_{0}}{2}\left\|v_{k}\right\|_{2}^{2} \rightarrow 0
$$

By virtue of (W4) and (2.10), one can get that

$$
\begin{aligned}
\sum_{n \in \Omega_{k}\left(R_{0}, \infty\right)} \frac{\left|W\left(n, u_{k}(n)\right)\right|}{\left|u_{k}(n)\right|^{2}}\left|v_{k}(n)\right|^{2} & \leq\left\|v_{k}\right\|_{\infty}^{2} \sum_{n \in \Omega_{k}\left(R_{0}, \infty\right)} \frac{\left|W\left(n, u_{k}(n)\right)\right|}{\left|u_{k}(n)\right|^{2}} \\
& \leq c_{1}\left[\sum_{n \in \Omega_{k}\left(R_{0}, \infty\right)} \widetilde{W}\left(n, u_{k}(n)\right)\right] \sum_{n \in \mathbb{Z}}\left|v_{k}(n)\right|^{2} \\
& \leq c_{1}\left[c+1-\sum_{n \in \Omega_{k}\left(0, R_{0}\right)} \widetilde{W}\left(n, u_{k}(n)\right)\right]\left\|v_{k}\right\|_{2}^{2} \\
& \leq c_{1}\left[c+1-\sum_{n \in \Omega_{k}\left(0, R_{0}\right)} g(n)\right]\left\|v_{k}\right\|_{2}^{2} \\
& \leq c_{1}\left[c+1+\sum_{n \in \mathbb{Z}}|g(n)|\right]\left\|v_{k}\right\|_{2}^{2} \rightarrow 0 .
\end{aligned}
$$

Combining (2.13) with (2.14), we have

$$
\begin{aligned}
\sum_{n \in \mathbb{Z}} \frac{\left|W\left(n, u_{k}(n)\right)\right|}{\left\|u_{k}\right\|^{2}}= & \sum_{n \in \Omega_{k}\left(0, R_{0}\right)} \frac{\left|W\left(n, u_{k}(n)\right)\right|}{\left|u_{k}(n)\right|^{2}}\left|v_{k}(n)\right|^{2} \\
& +\sum_{n \in \Omega_{k}\left(R_{0}, \infty\right)} \frac{\left|W\left(n, u_{k}(n)\right)\right|}{\left|u_{k}(n)\right|^{2}}\left|v_{k}(n)\right|^{2} \rightarrow 0
\end{aligned}
$$

which contradicts (2.11).

Set $A:=\{n \in \mathbb{Z}:|v(n)| \neq 0\}$. If $v \neq 0$, then $A \neq \emptyset$. For any $n \in A$, we have $\lim _{k \rightarrow+\infty}\left|u_{k}(n)\right|=$ $\infty$. Hence $A \subset \Omega_{k}\left(R_{0}, \infty\right)$ for large $k \in \mathbb{N}$, and it follows from (2.7), (W1), (W2) and Fatou's 
lemma that

$$
\begin{aligned}
0 & =\lim _{k \rightarrow \infty} \frac{c+o(1)}{\left\|u_{k}\right\|^{2}}=\lim _{k \rightarrow \infty} \frac{\Phi\left(u_{k}\right)}{\left\|u_{k}\right\|^{2}} \\
& =\lim _{k \rightarrow \infty}\left[\frac{1}{2}-\sum_{n \in \mathbb{Z}} \frac{W\left(n, u_{k}(n)\right)}{\left|u_{k}(n)\right|^{2}}\left|v_{k}(n)\right|^{2}\right] \\
& =\lim _{k \rightarrow \infty}\left[\frac{1}{2}-\sum_{n \in \Omega_{k}\left(0, R_{0}\right)} \frac{W\left(n, u_{k}(n)\right)}{\left|u_{k}(n)\right|^{2}}\left|v_{k}(n)\right|^{2}-\sum_{n \in \Omega_{k}\left(R_{0}, \infty\right)} \frac{W\left(n, u_{k}(n)\right)}{\left|u_{k}(n)\right|^{2}}\left|v_{k}(n)\right|^{2}\right] \\
& \leq \limsup _{k \rightarrow \infty}\left[\frac{1}{2}+\frac{c_{0}}{2} \sum_{n \in \mathbb{Z}}\left|v_{k}(n)\right|^{2}-\sum_{n \in \Omega_{k}\left(R_{0}, \infty\right)} \frac{W\left(n, u_{k}(n)\right)}{\left|u_{k}(n)\right|^{2}}\left|v_{k}(n)\right|^{2}\right] \\
& \leq \frac{1}{2}+\frac{c_{0} \gamma_{2}^{2}}{2}-\liminf _{k \rightarrow \infty} \sum_{n \in \Omega_{k}\left(R_{0}, \infty\right)} \frac{W\left(n, u_{k}(n)\right)}{\left|u_{k}(n)\right|}\left|v_{k}(n)\right|^{2} \\
& =\frac{1}{2}+\frac{c_{0} \gamma_{2}^{2}}{2}-\liminf _{k \rightarrow \infty} \sum_{n \in \mathbb{Z}} \frac{\left|W\left(n, u_{k}(n)\right)\right|}{\left|u_{k}(n)\right|^{2}}\left[\chi_{\Omega_{k}\left(R_{0}, \infty\right)}(n)\right]\left|v_{k}(n)\right|^{2} \\
& \leq \frac{1}{2}+\frac{c_{0} \gamma_{2}^{2}}{2}-\sum_{n \in \mathbb{Z}} \liminf _{k \rightarrow \infty} \frac{\left|W\left(n, u_{k}(n)\right)\right|}{\left|u_{k}(n)\right|^{2}}\left[\chi_{\Omega_{k}\left(R_{0}, \infty\right)}(n)\right]\left|v_{k}(n)\right|^{2} \\
& =-\infty,
\end{aligned}
$$

which is a contradiction. Thus $\left\{u_{k}\right\}$ is bounded in $E$.

Lemma 2.4 Under assumptions (L'), (W1), (W2) and (W4), any sequence $\left\{u_{k}\right\} \subset E$ satisfying (2.9) has a convergent subsequence in $E$.

Proof Lemma 2.3 implies that $\left\{u_{k}\right\}$ is bounded in $E$. Going if necessary to a subsequence, we can assume that $u_{k} \rightarrow u$ in $E$. By Lemma 2.2, $u_{k} \rightarrow u$ in $l^{q}\left(\mathbb{Z}, \mathbb{R}^{\mathcal{N}}\right)$ for $2 \leq q<\infty$, and $u_{k}(n) \rightarrow u(n)$ for all $n \in \mathbb{Z}$. By $\left(\mathrm{L}^{\prime}\right)$, there exists an integer $N \in \mathbb{N}$ such that

$$
\left|u_{k}(n)\right|^{2} \leq \frac{1}{\min _{|s| \geq|n|} l(s)} \sum_{|s| \geq|n|} l(s)\left|u_{k}(s)\right|^{2} \leq \frac{1}{\min _{|s| \geq|n|} l(s)}\left\|u_{k}\right\|^{2}<R_{0}^{2}, \quad|n| \geq N .
$$

It is easy to see that

$$
\sum_{|n| \leq N}\left|\nabla W\left(n, u_{k}(n)\right)-\nabla W(n, u(n))\right|\left|u_{k}(n)-u(n)\right| \rightarrow 0, \quad k \rightarrow+\infty
$$

Next, we prove that

$$
\sum_{|n|>N}\left|\nabla W\left(n, u_{k}(n)\right)-\nabla W(n, u(n))\right|\left|u_{k}(n)-u(n)\right| \rightarrow 0, \quad k \rightarrow+\infty .
$$

If (2.18) is not true, then there exist a constant $\varepsilon_{0}>0$ and a subsequence $\left\{u_{k_{i}}\right\}$ such that

$$
\sum_{|n|>N}\left|\nabla W\left(n, u_{k_{i}}(n)\right)-\nabla W(n, u(n))\right|\left|u_{k_{i}}(n)-u(n)\right| \geq \varepsilon_{0}, \quad \forall i \in \mathbb{N} .
$$


Since $u_{k} \rightarrow u$ in $l^{2}\left(\mathbb{Z}, \mathbb{R}^{\mathcal{N}}\right)$, passing to a subsequence if necessary, it can be assumed that $\sum_{i=1}^{\infty}\left\|u_{k_{i}}-u\right\|_{2}^{2}<+\infty$. Set

$$
w(n)=\left[\sum_{i=1}^{\infty}\left|u_{k_{i}}(n)-u(n)\right|^{2}\right]^{1 / 2}, \quad n \in \mathbb{Z} .
$$

Then $w \in l^{2}(\mathbb{Z}, \mathbb{R})$. From (2.16), (2.20) and (W1), one has

$$
\begin{aligned}
\mid \nabla & W\left(n, u_{k_{i}}(n)\right)-\nabla W(n, u(n))|| u_{k_{i}}(n)-u(n) \mid \\
& \leq\left(\left|\nabla W\left(n, u_{k_{i}}(n)\right)\right|+|\nabla W(n, u(n))|\right)\left(\left|u_{k_{i}}(n)\right|+|u(n)|\right) \\
& \leq c_{0}\left(\left|u_{k_{i}}(n)\right|+|u(n)|\right)^{2} \\
& \leq 2 c_{0}\left(\left|u_{k_{i}}(n)\right|^{2}+|u(n)|^{2}\right) \\
& \leq 8 c_{0}\left(\left|u_{k_{i}}(n)-u(n)\right|^{2}+|u(n)|^{2}\right) \\
& \leq 8 c_{0}\left([w(n)]^{2}+|u(n)|^{2}\right) \\
& :=h(n), \quad \forall i \in \mathbb{N},|n| \geq N
\end{aligned}
$$

and

$$
\begin{aligned}
\sum_{|n|>N} h(n) & =8 c_{0} \sum_{|n|>N}\left([w(n)]^{2}+|u(n)|^{2}\right) \\
& \leq 8 c_{0}\left(\|w\|_{2}^{2}+\|u\|_{2}^{2}\right)<+\infty .
\end{aligned}
$$

Since $u_{k_{i}}(n) \rightarrow u(n)$ for all $n \in \mathbb{Z}$, then by (2.21), (2.22) and Lebesgue's dominated convergence theorem, we have

$$
\lim _{i \rightarrow \infty} \sum_{|n|>N}\left|\nabla W\left(n, u_{k_{i}}(n)\right)-\nabla W(n, u(n))\right|\left|u_{k_{i}}(n)-u(n)\right|=0,
$$

which contradicts (2.19). Hence (2.18) holds. Combining (2.17) with (2.18), one has

$$
\sum_{n \in \mathbb{Z}}\left|\nabla W\left(n, u_{k}(n)\right)-\nabla W(n, u(n))\right|\left|u_{k}(n)-u(n)\right| \rightarrow 0, \quad n \rightarrow \infty .
$$

Observe that

$$
\begin{aligned}
\left\|u_{k}-u\right\|^{2}= & \left\langle\Phi^{\prime}\left(u_{k}\right)-\Phi^{\prime}(u), u_{k}-u\right\rangle \\
& +\sum_{n \in \mathbb{Z}}\left(\nabla W\left(n, u_{k}(n)\right)-\nabla W(n, u(n)), u_{k}(n)-u(n)\right) .
\end{aligned}
$$

It is clear that

$$
\left\langle\Phi^{\prime}\left(u_{k}\right)-\Phi^{\prime}(u), u_{k}-u\right\rangle \rightarrow 0, \quad n \rightarrow \infty
$$

From (2.23), (2.24) and (2.25), we have $\left\|u_{k}-u\right\| \rightarrow 0, n \rightarrow \infty$. 
Lemma 2.5 Under assumptions (L'), (W1) and (W5), any sequence $\left\{u_{k}\right\} \subset E$ satisfying (2.9) has a convergent subsequence in $E$.

Proof First, we prove that $\left\{u_{k}\right\}$ is bounded in $E$. To this end, arguing by contradiction, suppose that $\left\|u_{k}\right\| \rightarrow \infty$. Let $v_{k}=u_{k} /\left\|u_{k}\right\|$. Then $\left\|v_{k}\right\|=1$ and $\left\|v_{k}\right\|_{q} \leq \gamma_{q}\left\|v_{k}\right\|=\gamma_{q}$ for $2 \leq q \leq \infty$. By (2.7), (2.8), (2.9) and (W5), one has

$$
\begin{aligned}
c+1 & \geq \Phi\left(u_{k}\right)-\frac{1}{\mu}\left\langle\Phi^{\prime}\left(u_{k}\right), u_{k}\right\rangle \\
& =\frac{\mu-2}{2 \mu}\left\|u_{k}\right\|^{2}+\sum_{n \in \mathbb{Z}}\left[\frac{1}{\mu}\left(\nabla W\left(n, u_{k}(n)\right), u_{k}(n)\right)-W\left(n, u_{k}(n)\right)\right] \\
& \geq \frac{\mu-2}{2 \mu}\left\|u_{k}\right\|^{2}-\frac{\varrho}{\mu}\left\|u_{k}\right\|_{2}^{2} \quad \text { for large } k \in \mathbb{N},
\end{aligned}
$$

which implies

$$
1 \leq \frac{2 \varrho}{\mu-2} \limsup _{k \rightarrow \infty}\left\|v_{k}\right\|_{2}^{2}
$$

Passing to a subsequence, we may assume that $v_{k} \rightarrow v$ in $E$, then by Lemma 2.1, $v_{k} \rightarrow$ $v$ in $l^{q}\left(\mathbb{Z}, \mathbb{R}^{\mathcal{N}}\right), 2 \leq q<\infty$, and $v_{k}(n) \rightarrow v(n)$ for all $n \in \mathbb{Z}$. Hence, it follows from (2.27) that $v \neq 0$. Analogous to the proof of (2.15), we can deduce a contradiction. Thus, $\left\{u_{k}\right\}$ is bounded in $E$. The rest of the proof is the same as the one in Lemma 2.4.

Lemma 2.6 Under assumptions (L'), (W1) and (W2), for any finite-dimensional subspace $\tilde{E} \subset E$, there holds

$$
\Phi(u) \rightarrow-\infty, \quad\|u\| \rightarrow \infty, u \in \tilde{E} .
$$

Proof Arguing indirectly, assume that for some sequence $\left\{u_{k}\right\} \subset \tilde{E}$ with $\left\|u_{k}\right\| \rightarrow \infty$, and there is $M_{2}>0$ such that $\Phi\left(u_{k}\right) \geq-M_{2}$ for all $k \in \mathbb{N}$. Set $v_{k}=u_{k} /\left\|u_{k}\right\|$, then $\left\|v_{k}\right\|=1$. Passing to a subsequence, we may assume that $v_{k} \rightarrow v$ in $E$. Since $\tilde{E}$ is finite-dimensional, then $v_{k} \rightarrow$ $v \in \tilde{E}$ in $E, v_{k}(n) \rightarrow v(n)$ for all $n \in \mathbb{Z}$, and so $\|v\|=1$. Hence, we can deduce a contradiction in the same way as (2.15).

Corollary 2.7 Under assumptions (L'), (W1) and (W2), for any finite-dimensional subspace $\tilde{E} \subset E$, there is $R=R(\tilde{E})>0$ such that

$$
\Phi(u) \leq 0, \quad \forall u \in \tilde{E},\|u\| \geq R .
$$

Let $\left\{e_{j}\right\}$ is an orthonomormal basis of $E$ and define $X_{j}=\mathbb{R} e_{j}$,

$$
Y_{k}=\bigoplus_{j=1}^{k} X_{j}, \quad Z_{k}=\bigoplus_{j=k+1}^{\infty} X_{j}, \quad k \in \mathbb{N} .
$$

Lemma 2.8 Under assumption ( $\left.\mathrm{L}^{\prime}\right)$, for $2 \leq q<\infty$,

$$
\beta_{k}(q):=\sup _{u \in Z_{k},\|u\|=1}\|u\|_{q} \rightarrow 0, \quad k \rightarrow \infty .
$$


Proof Since the embedding from $E$ into $l^{q}\left(\mathbb{Z}, \mathbb{R}^{\mathcal{N}}\right)$ is compact for $2 \leq q<\infty$, then Lemma 2.8 can be proved in a similar way as [22, Lemma 3.8].

Applying Lemma 2.8, we can choose an integer $m \geq 1$ such that

$$
\|u\|_{2}^{2} \leq \frac{1}{2 c_{0}}\|u\|^{2}, \quad \forall u \in Z_{m}
$$

Lemma 2.9 Under assumptions (L') and (W1), there exist constants $\rho, \alpha>0$ such that $\left.\Phi\right|_{\partial B_{\rho} \cap Z_{m}} \geq \alpha$.

Proof If $\|u\|=R_{0}$, then $\|u\|_{\infty} \leq R_{0}$. Hence, it follows from (W1) that

$$
|W(n, u(n))| \leq \frac{c_{0}}{2}|u(n)|^{2}, \quad \forall u \in E,\|u\|=R_{0}
$$

By (2.7), (2.32) and (2.33), we have

$$
\begin{aligned}
\Phi(u) & =\frac{1}{2}\|u\|^{2}-\sum_{n \in \mathbb{Z}} W(n, u(n)) \geq \frac{1}{2}\|u\|^{2}-\frac{c_{0}}{2} \sum_{n \in \mathbb{Z}}|u(n)|^{2} \\
& =\frac{1}{2}\|u\|^{2}-\frac{c_{0}}{2}\|u\|_{2}^{2} \geq \frac{1}{4}\|u\|^{2} \\
& =\frac{1}{4} R_{0}^{2}:=\alpha, \quad \forall u \in Z_{m},\|u\|=R_{0}:=\rho .
\end{aligned}
$$

We say that $I \in C^{1}(X, \mathbb{R})$ satisfies $(C)_{c}$-condition if any sequence $\left\{u_{k}\right\}$ such that

$$
I\left(u_{k}\right) \rightarrow c, \quad\left\|I^{\prime}\left(u_{k}\right)\right\|\left(1+\left\|u_{k}\right\|\right) \rightarrow 0
$$

has a convergent subsequence.

Lemma 2.10 (Bartolo, Benci and Fortunato [23]) Let $X$ be an infinite-dimensional Banach space, $X=Y \oplus Z$, where $Y$ is finite-dimensional. If $I \in C^{1}(X, \mathbb{R})$ satisfies $(C)_{c}$-condition for all $c>0$, and

(I1) $I(0)=0, I(-u)=I(u)$ for all $u \in X$;

(I2) there exist constants $\rho, \alpha>0$ such that $\left.\Phi\right|_{\partial B_{\rho} \cap Z} \geq \alpha$;

(I3) for any finite-dimensional subspace $\tilde{X} \subset X$, there is $R=R(\tilde{X})>0$ such that $I(u) \leq 0$ on $\tilde{X} \backslash B_{R}$;

then I possesses an unbounded sequence of critical values.

\section{Proofs of the main results}

By (L), there exists a constant $a_{0}>0$ such that

$$
\left(\left(L(n)+2 a_{0} I_{N}\right) x, x\right) \geq|x|^{2}, \quad \forall(n, x) \in \mathbb{Z} \times \mathbb{R}^{\mathcal{N}}
$$

Let $\bar{L}(n):=L(n)+2 a_{0} I_{\mathcal{N}}$ and $\bar{W}(n, x)=W(n, x)+a_{0}|x|^{2}$. Then it is easy to verify the following lemma. 
Lemma 3.1 $u \in S$ is a solution of system (1.1) if and only if it is a solution of the following system

$$
\triangle[p(n) \triangle u(n-1)]-\bar{L}(n) u(n)+\nabla \bar{W}(n, u(n))=0 .
$$

Proof of Theorem 1.1 Let $X=E, Y=Y_{m}$, and let $Z=Z_{m}$. Obviously, $\bar{W}$ satisfies (W1), (W2), (W3) and (W4). By Lemmas 2.3, 2.4, 2.9 and Corollary 2.7, all conditions of Lemma 2.10 are satisfied. Thus, system (3.2) possesses infinitely many nontrivial solutions. By Lemma 3.1, system (1.1) also possesses infinitely many nontrivial solutions.

Proof of Theorem 1.2 Let $X=E, Y=Y_{m}$, and let $Z=Z_{m}$. Obviously, $\bar{W}$ satisfies (W1), (W2), (W3) and (W5). The rest of the proof is the same as that of Theorem 1.1 by using Lemma 2.5 instead of Lemmas 2.3 and 2.4.

\section{Competing interests}

The authors declare that they have no competing interests.

\section{Authors' contributions}

All authors jointly worked on the results, and they read and approved the final manuscript.

\section{Acknowledgements}

The authors would like to express their thanks to the referees for their helpful suggestions. This work is partially supported by the NNSF (No. 11171351) of China and supported by the Hunan Provincial Natural Science Foundation of China (No. 11JJ2005).

\section{Received: 19 April 2013 Accepted: 24 July 2013 Published: 8 August 2013}

\section{References}

1. Agarwal, RP: Difference Equations and Inequalities: Theory, Methods and Applications, 2nd edn. Dekker, New York (2000)

2. Ahlbran, CD, Peterson, AC: Discrete Hamiltonian Systems: Difference Equations, Continued Fraction and Riccati Equations. Kluwer Academic, Dordrecht (1996)

3. Chen, WX, Yang, MB, Ding, YH: Homoclinic orbits of first order discrete Hamiltonian systems with super linear terms. Sci. China, Math. 54, 2583-2596 (2011)

4. Coti Zelati, V, Ekeland, I, Sere, E: A variational approach to homoclinic orbits in Hamiltonian systems. Math. Ann. 288(1), 133-160 (1990)

5. Coti Zelati, V, Rabinowitz, PH: Homoclinic orbits for second order Hamiltonian systems possessing superquadratic potentials. J. Am. Math. Soc. 4, 693-727 (1991)

6. Ding, YH: Existence and multiplicity results for homoclinic solutions to a class of Hamiltonian systems. Nonlinear Anal. 25(11), 1095-1113 (1995)

7. Rabinowitz, PH: Homoclinic orbits for a class of Hamiltonian systems. Proc. R. Soc. Edinb. A 114(1-2), 33-38 (1990)

8. Rabinowitz, PH, Tanaka, K: Some results on connecting orbits for a class of Hamiltonian systems. Math. Z. 206(3), 473-499 (1991)

9. Tang, XH, Lin, XY: Existence of infinitely many homoclinic orbits in Hamiltonian systems. Proc. R. Soc. Edinb. A 141, 1103-1119 (2011)

10. Wong, JSW: On the generalized Emden-Fowler equation. SIAM Rev. 17, 339-360 (1975)

11. Deng, XQ, Cheng, G, Shi, HP: Subharmonic solutions and homoclinic orbits of second order discrete Hamiltonian systems with potential changing sign. Comput. Math. Appl. 58, 1198-1206 (2009)

12. Ma, M, Guo, ZM: Homoclinic orbits and subharmonics for nonlinear second order difference equations. Nonlinear Anal. 67, 1737-1745 (2007)

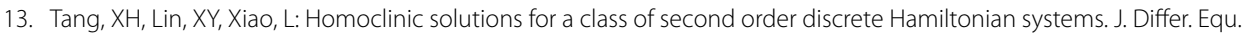
Appl. 16, 1257-1273 (2010)

14. Ma, M, Guo, ZM: Homoclinic orbits for second order self-adjoint difference equations. J. Math. Anal. Appl. 323(1), 513-521 (2006)

15. Lin, $X Y$, Tang, $X H$ : Existence of infinitely many homoclinic orbits in discrete Hamiltonian systems. J. Math. Anal. Appl. 373, 59-72 (2011)

16. Tang, $\mathrm{XH}$, Lin, $\mathrm{XY}$ : Homoclinic solutions for a class of second order discrete Hamiltonian systems. Acta Math. Sin. 28 609-622 (2012)

17. Tang, $\mathrm{XH}$, Lin, $\mathrm{XY}$ : Infinitely many homoclinic orbits for discrete Hamiltonian systems with subquadratic potential. J. Differ. Equ. Appl. 19, 796-813 (2012)

18. Yu, JS, Shi, HP, Guo, ZM: Homoclinic orbits for nonlinear difference equations containing both advance and retardation. J. Math. Anal. Appl. 352, 799-806 (2009) 
19. Zhou, Z, Yu, JS, Chen, Y: Homoclinic solutions in periodic difference equations with saturable nonlinearity. Sci. China, Math. 54, 83-93 (2011)

20. Tang, XH, Lin, XY: Existence and multiplicity of homoclinic solutions for second-order discrete Hamiltonian systems with subquadratic potential. J. Differ. Equ. Appl. 17, 1617-1634 (2011)

21. Deng, XQ, Cheng, G: Homoclinic orbits for second order discrete Hamiltonian systems with potential changing sign. Acta Appl. Math. 103, 301-314 (2008). doi:10.1007/s10440-008-9237-z

22. Willem, M: Minimax Theorems. Birkhäuser, Boston (1996)

23. Bartolo, T, Benci, V, Fortunato, D: Abstract critical point theorems and applications to some nonlinear problems with strong resonance at infinity. Nonlinear Anal. 7, 241-273 (1983)

doi:10.1186/1687-1847-2013-242

Cite this article as: Tang and Chen: Infinitely many homoclinic orbits for a class of discrete Hamiltonian systems. Advances in Difference Equations 2013 2013:242.

\section{Submit your manuscript to a SpringerOpen ${ }^{0}$ journal and benefit from:}

- Convenient online submission

- Rigorous peer review

- Immediate publication on acceptance

- Open access: articles freely available online

- High visibility within the field

- Retaining the copyright to your article 